'Servicio de medicina interna, Sección nefrología, Hospital Naval A. Nef, Viña del Mar, Chile. ${ }^{2}$ Servicio de medicina interna, Sección Nefrología, Hospital Dr. Gustavo Fricke, Viña del Mar, Chile.

'Departamento de medicina, Escuela de medicina, Universidad de Valparaíso, Valparaíso, Chile. ${ }^{4}$ Servicio de radiología, Hospital Naval A. Nef, Viña del Mar, Chile.

Recibido el 30 de septiembre de 2015, aceptado el 2 de diciembre de 2015.

Correspondencia a: Dr. Jorge Vega Stieb 5 Norte 1035 Viña del Mar, Chile. Teléfono 56-32-2974237 jvegastieb@gmail.com

\section{Tratamiento con sirolimus y prednisona en enfermedad de Erdheim-Chester con compromiso cardiaco. Caso clínico}

\author{
JORGE VEGA ${ }^{1,2,3}$, MARIO SANTAMARINA ${ }^{4}$
}

\section{Sirolimus and prednisone for the treatment of Erdheim-Chester disease with cardiac involvement. Report of one case}

Erdheim-Chester disease (ECD) is a rare non-Langerhans histiocytic disorder. We report a 76-years-old man who suffered a cardiac tamponade secondary to ECD. A pericardial window was made and during the operation the surgeons observed that the myocardium was diffusely infiltrated. Twenty-eight months before, ECD was clinically diagnosed and prednisone and methotrexate were prescribed. Due to disease progression which culminated in the cardiac tamponade, methotrexate was changed to sirolimus aiming to obtain plasma levels between 5-8 $\mathrm{ng} / \mathrm{ml}$. This treatment stabilized his cardiac function allowing a survival of 52 months after its initiation, with fewer side effects.

(Rev Med Chile 2016; 144: 394-400)

Key words: Cardiac Tamponade; Erdheim-Chester Disease; Heart Failure; Sirolimus.
L a enfermedad de Erdheim-Chester (EEC) es una variedad rara de histiocitosis, habiéndose comunicado alrededor de 500 casos $^{1}$. Frecuentemente existe compromiso cardiovascular, que puede ser asintomático y detectarse en RM y/o scanner ${ }^{2-4}$. Las anormalidades más frecuentes son: a) recubrimiento de la aorta por un manguito de tejido-pudiendo comprometer sus ramas; b) infiltración pericárdica, que puede manifestarse por pericarditis, derrame pericárdico, taponamiento cardiaco o pericarditis constrictiva. Menos frecuentemente se observa: c) infiltración miocárdica-pudiendo originar insuficiencia cardiaca; d) infiltración de la aurícula derecha semejando un tumor cardiaco; e) infiltración de los sulcus aurículo-ventriculares, septum interauricular y lecho peri-coronario-pudiendo originar infartos; f) valvulopatías aórtica y mitral-produciendo regurgitación; g) anormalidades de la conducción 0 arritmias ${ }^{3-6}$. La falla cardiaca inducida por la fibrosis miocárdica y/o el compromiso pericárdico son las principales causas de muerte ${ }^{7}$.

El interferón-alfa (IFN- $\alpha$ ) ha sido la droga más utilizada $^{8,9}$, pero frecuentemente es poco efectiva, mal tolerada o se desarrolla resistencia durante su uso en los pacientes con compromiso de SNC o cardiovascular'. En ellos se han ensayado drogas antineoplásicas, biológicas e inmunosupreso$\operatorname{ras}^{3,7,10-21}$

Comunicamos un caso de insuficiencia cardiaca (ICC) y signos de taponamiento por infiltración del corazón por EEC, que tuvo una larga sobrevivencia bajo terapia con sirolimus.

\section{Caso clínico}

Hombre de 76 años. Se le efectuó una ventana pericárdica en abril de 2010 por derrame con signos de taponamiento (Figura 1). El pericardio 


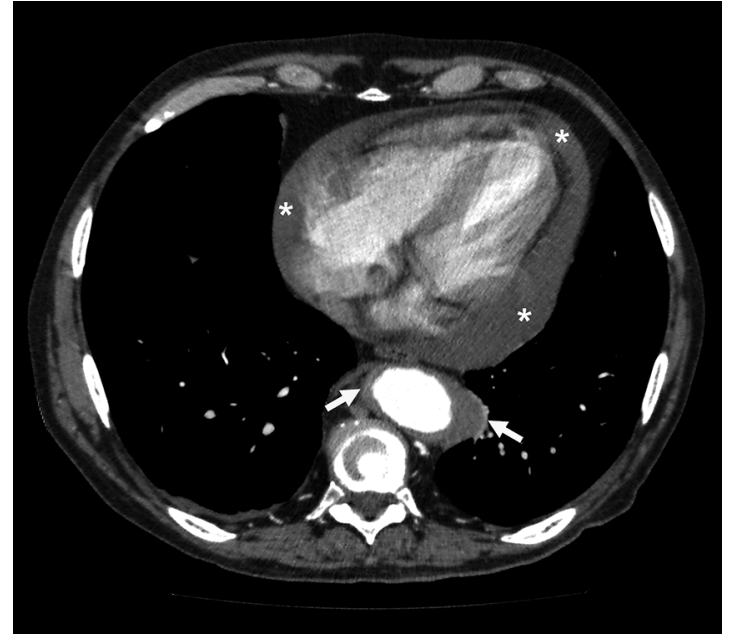

Figura 1. Corte transversal del tórax a nivel cardiaco. Imagen cardiaca rodeada de líquido pericárdico (asteriscos). La aorta está cubierta de un tejido que se refuerza con el medio de contraste (flechas blancas).

visceral estaba engrosado, duro e irregular con aspecto de "maní confitado". Se drenó $1.200 \mathrm{ml}$ de líquido que contenía abundantes histiocitos. $\mathrm{La}$ biopsia del pericardio parietal mostró infiltración por histiocitos de citoplasma granular y espumoso cuyo análisis inmuno-histoquímico mostró po- sitividad para CD68 y negatividad para CD1a y proteína S100, característica de EEC.

Veintiocho meses antes se había efectuado el diagnóstico clínico de EEC a raíz de compromiso del estado general, anemia, VHS elevada, hemoptisis, presencia de un tejido que a modo de manguito envolvía la aorta torácica y abdominal produciendo compresión extrínseca de las arterias mesentéricas, tronco celíaco y renales (requirieron la instalación de stents), esplenomegalia y la presencia de osteoesclerosis en tibias y fémures (considerado signo patognomónico de esa enfermedad). Existían además signos radiológicos típicos de condrocalcinosis en las rodillas. Desde entonces se encontraba en tratamiento con metotrexato (MTX) $10 \mathrm{mg} / \mathrm{semanales} \mathrm{y} \mathrm{prednisona} 10 \mathrm{mg} /$ día.

La historia de este paciente hasta el momento del taponamiento cardiaco fue publicada previamente en esta misma revista ${ }^{22}$.

Posterior al drenaje, su ICC mejoró parcialmente quedando en capacidad funcional II-III (NYHA). Días después presentó una fibrilación auricular que revirtió con amiodarona.

En junio de 2010, un scanner mostró un tejido que englobaba las arterias coronarias y engrosamiento pericárdico con escaso derrame (Figura 2). En vista de la progresión de la enfermedad bajo

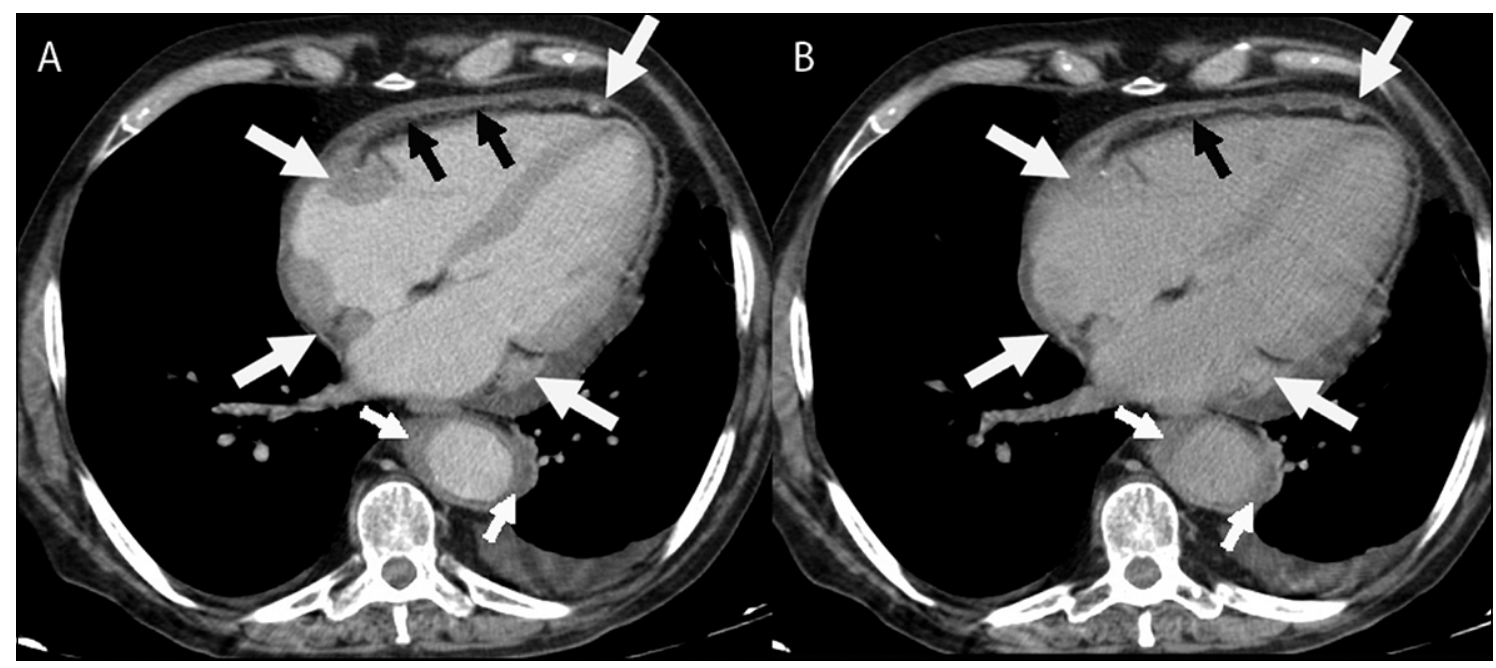

Figura 2. A) Scanner con contraste ev. 70 segundos después de la administración del contraste ev. Tejido de densidad de partes blandas, con realce, que engloba las arterias coronarias en su totalidad (flechas blancas grandes) y la aorta torácica descendente (flechas blancas pequeñas). Engrosamiento pericárdico (flechas negras), asociado a mínimo derrame. B) Scanner con contraste ev. 240 segundos después de la administración del contraste ev. Este tejido de densidad de partes blandas que engloba las arterias coronarias (flechas blancas grandes) y la aorta torácica descendente (flechas blancas pequeñas) presenta marcado realce tardío que prácticamente se solapa con el contraste vascular y cardíaco. Engrosamiento pericárdico (flechas negras), asociado a mínimo derrame. 
MTX y las publicaciones que comunicaban que cuando existía compromiso cardiaco la terapia de elección de la EEC con interferón a menudo era ineficiente en el control de la enfermedad, se prescribió sirolimus $(2 \mathrm{mg} /$ día $)$ asociado a prednisona $10 \mathrm{mg} /$ día, dada una comunicación reciente del resultado exitoso en algunos enfermos con compromiso cardiaco ${ }^{23}$. El paciente aceptó su utilización. La tolerancia fue buena, salvo ocasionales episodios de diarrea. En los meses siguientes sirolimus se redujo a $1 \mathrm{mg} /$ día obteniendo niveles terapéuticos (5 a $8 \mathrm{ng} / \mathrm{ml}$ ). Recibía además anagrelide (por trombocitemia esencial), valsaltan, furosemide, hidroclorotiazida y citalopram.

En septiembre de 2010 un ecocardiograma mostró ausencia de derrame pericárdico, función sistólica de ventrículo izquierdo normal con anormalidades en la relajación (Tabla 1 ). A raíz de anemización se efectuó panendoscopia que reveló un pólipo hiperplástico yuxtacardial con evidencias de sangrado. Fue tratado localmente y con omeprazol.

En noviembre de 2010 se diagnosticó hipogonadismo hipogonadotrófico e hipotiroidismo secundario a hipopituitarismo (Tabla 2). La resonancia magnética mostró anormalidades hipofisiarias compatibles con histiocitosis. No existían otras lesiones en el sistema nervioso central. Se prescribió testosterona y tiroxina, con mejoría significativa del estado general. Entre el 2011 y 2013 no hubo variaciones en su función cardiaca, con excepción de un episodio de edema pulmonar agudo. Este se presentó en asociación a una elevación brusca de peso por ingesta excesiva de sal en la dieta y la reducción en la dosis de furosemide prescrita. Al examen físico había disnea de reposo, ortopnea, sibilancias, gran ingurgitación yugular, galope y crépitos bilaterales difusos en ambos campos pulmonares. Se trató en su domicilio con dosis abundantes de furosemida y nitrato de isosorbide, volviendo en pocos días a su condición basal. Nunca presentó angina a pesar del compromiso peri-coronario.

Durante los primeros 4 años de tratamiento con sirolimus no hubo efectos secundarios relevantes salvo diarrea intermitente. Existió leve hipertrigliceridemia, función renal inestable, proteinuria mínima y alteraciones hematológicas atribuidas a su trombocitemia y al anagrelide (Tabla 2).

Tabla 1

\begin{tabular}{|c|c|c|c|}
\hline Mediciones ecocardiográficas & $\begin{array}{l}6 \text { meses antes } \\
\text { de la ventana } \\
\text { pericárdica } \\
\text { Sin sirolimus }\end{array}$ & $\begin{array}{c}5 \text { meses después } \\
\text { de la ventana } \\
\text { pericárdica } \\
2 \text { meses con sirolimus }\end{array}$ & $\begin{array}{c}46 \text { meses después de } \\
\text { la ventana } \\
\text { pericárdica }\end{array}$ \\
\hline Pared posterior (6-11 mm) & 10 & 11 & 10 \\
\hline Septum ventricular (6-11 mm) & 13 & 14 & 11 \\
\hline Dimensión diastólica VI (35-57mm) & 49 & 51 & 55 \\
\hline Dimensión sistólica VI (25-41mm) & 32 & 34 & 32 \\
\hline Aorta diámetro diastólico (19-40 mm) & 40 & 38 & 40 \\
\hline Aurícula izquierda (19-40 mm) & 36 & 41 & 42 \\
\hline Fracción de eyección (65-75\%) & & 62 & 73 \\
\hline Válvula aórtica & Reflujo leve & Reflujo moderado & No tiene \\
\hline Válvula mitral & Normal & Normal & Normal \\
\hline Disfunción diastólica de V. izquierdo & & Grado II & Grado II \\
\hline Aurícula derecha & & & Normal \\
\hline Ventrículo derecho & Normal & Normal & Normal \\
\hline Presión arterial pulmonar estimada & & Normal & No estimada \\
\hline Pericardio & Normal, sin derrame & Mínimo derrame & Normal, sin derrame \\
\hline
\end{tabular}


Tabla 2

\begin{tabular}{|c|c|c|c|c|c|}
\hline & Año 2010 & Año 2011 & Año 2012 & Año 2013 & Año 2014 \\
\hline \multicolumn{6}{|l|}{ Función renal } \\
\hline Uremia (mg/dl) & $47-57$ & $64-168$ & $78-183$ & 84-102 & $75-117$ \\
\hline Creatininemia (mg/dl) & $1,16-1,91$ & $1,65-3,27$ & $1,79-3,28$ & $1,87-2,03$ & $1,79-2,05$ \\
\hline Proteinuria & Negativa & Negativa a $(+)$ & Negativa a $(+)$ & Negativa & Negativa a $(+)$ \\
\hline \multicolumn{6}{|l|}{ Perfil hepático } \\
\hline Bilirrubina total (mg/dl) & $0,31-0,35$ & $0,25-0,51$ & $0,33-0,37$ & $0,14-0,15$ & $0,36-0,44$ \\
\hline Fosfatasas alcalinas (U/L) VN: 40-129 & $48-64$ & $45-54$ & $48-69$ & $49-65$ & $54-62$ \\
\hline Proteinemia (g/dl) & $5,6-6,2$ & $5,6-6,3$ & $5,8-6,4$ & $6,4-6,6$ & $6,1-6,4$ \\
\hline Albuminemia (g/dl) & $3,3-3,4$ & $3,2-3,7$ & $3,44,0$ & $3,7-3,9$ & $3,7-3,8$ \\
\hline Globulinas (g/dl) & $2,3-2,8$ & $2,2-2,8$ & $2,3-2,5$ & $2,6-2,7$ & $2,4-2,6$ \\
\hline SGOT (U/L) & $5-6$ & $5-9$ & $5-6$ & $5-6$ & $5-7$ \\
\hline SGPT (U/L & $5-6$ & $5-47$ & 5 & $5-6$ & 5 \\
\hline GGTP (U/L) & $17-22$ & 20 & & & \\
\hline LDH (U/L) VN:135-225 & $158-246$ & 153-192 & $163-246$ & $244-279$ & $213-266$ \\
\hline \multicolumn{6}{|l|}{ Perfil lipídico } \\
\hline Colesterol total (mg/dl) & $132-196$ & $158-201$ & $162-202$ & $202-213$ & $197-209$ \\
\hline Triglicéridos (mg/dl) & $115-204$ & $146-222$ & $137-288$ & $130-219$ & $201-218$ \\
\hline \multicolumn{6}{|l|}{ Perfil hematológico } \\
\hline Hematocrito (\%) & $26,1-34,8$ & $28,9-36,8$ & $32,7-39,0$ & $36,1-37,8$ & $31,1-34,7$ \\
\hline Hemoglobina (g/dl) & $8,0-10,6$ & $8,9-11,4$ & $9,5-11,2$ & $10,9-11,6$ & $9,3-10,8$ \\
\hline Leucocitos $\left(x \mathrm{~mm}^{3}\right)$ & $\begin{array}{c}10.900- \\
19.900\end{array}$ & $\begin{array}{l}11.500- \\
17.430\end{array}$ & $\begin{array}{l}13.500- \\
22.510\end{array}$ & $\begin{array}{c}14.060- \\
17.510\end{array}$ & $\begin{array}{c}13.840- \\
15,180\end{array}$ \\
\hline Plaquetas $\left(\mathrm{x} \mathrm{mm}^{3}\right)$ & $\begin{array}{l}301.000- \\
642.000\end{array}$ & $\begin{array}{l}406.000- \\
682.000\end{array}$ & $\begin{array}{l}335.000- \\
701.000\end{array}$ & $\begin{array}{l}713.000- \\
912.000\end{array}$ & $\begin{array}{l}389.000- \\
587.000\end{array}$ \\
\hline VHS (mm/hora) & $28-110$ & $50-119$ & $25-70$ & $25-61$ & $26-50$ \\
\hline Ferritina (ng/ml) & 194 & 416 & 352 & & \\
\hline \multicolumn{6}{|l|}{ Hormonas } \\
\hline TSH (uUl/ml) VN: 0,47-4,68 & $0,56-0,09$ & 0,52 & 0,32 & $0,60-0,65$ & 0,70 \\
\hline $\mathrm{T} 4$ (ug/dl) VN: 5,1-14,0 & 5,93 & & & & \\
\hline T3 (ng/ml) VN: 0,8-2,0 & 0,44 & & & & \\
\hline T4 libre (pmol/L) VN: 10-28 & & 18,75 & & & \\
\hline $\mathrm{FSH}(\mathrm{mUl} / \mathrm{ml}) \mathrm{VN}: 1,6-12,4$ & 1,85 & & & & \\
\hline $\mathrm{LH}(\mathrm{mUl} / \mathrm{ml}) \mathrm{VN}: 1,7-8,6$ & 0,47 & & & & \\
\hline Testosterona total $(\mathrm{ng} / \mathrm{ml}) \mathrm{VN}: 2,8-8,0$ & 0,03 & & & & \\
\hline Prolactina (ng/ml) VN: 3,7-15,2 & 11,73 & & & & \\
\hline IGF-1 (ng/ml) VN: 59-177 & & 110 & & & \\
\hline
\end{tabular}

A comienzos de 2014, apareció una úlcera en la cara lateral del cuello que se expandió rápidamente, diagnosticándose un carcinoma espinocelular. Un ecocardiograma no mostró progresión de su cardiopatía (Tabla 1). En octubre de 2014 se resecó dicha lesión con anestesia general. En el postoperatorio inmediato hubo sangrado profuso del área operada con hipotensión arterial, requiriendo la transfusión de varias unidades de glóbulos rojos y plaquetas. Tuvo que ser reintervenido para controlar la hemorragia en 2 oportunidades, lo que se logró después de una nueva transfusión de plaquetas. El aporte generoso de volumen y hemoderivados se complicó de edema pulmonar agudo. Se conectó a ventilación mecánica y se administró furosemide, noradrenalina y dobutamina. A pesar 
de ello desarrolló hipotensión arterial persistente y anuria. Su familia, considerando su patología de base, no aceptó la proposición de monitoreo hemodinámico invasivo ni de diálisis con ultrafiltración. El paciente falleció dos días después en shock cardiogénico a los 80 años, 82 meses después del diagnóstico clínico de EEC, 54 meses después del diagnóstico histológico y 52 meses después del inicio de sirolimus.

\section{Discusión}

La EEC ha sido considerada una patología inflamatoria en que existe una respuesta inmune sistémica mediada por Th-1. Los histiocitos circulantes y del sitio de la lesión expresan un patrón de citokinas y quemokinas caracterizado por la elevación de interferón $\alpha$, interleukina 6 , interleukina 12 y MCP-13,4,10.

En los últimos años ha aparecido evidencia que sugiere que la EEC es también de naturaleza monoclonal. Diversas series han encontrado una mutación en BRAF (BRAF ${ }^{\mathrm{V} 600 \mathrm{E}}$ ) entre $50 \%$ y $100 \%$ de los pacientes, dependiendo del método de detección empleado ${ }^{21}$. Esta es una mutación activante del proto-oncogen BRAF que está presente en varios tumores humanos. Las mutaciones en este gen resultan en una señal que lleva a una excesiva proliferación celular ${ }^{18}$.

Actualmente la EEC puede definirse como un desorden monoclonal con una hiperactivación de la señal de protein-kinasa activada por mitógenos en el cual el ambiente inflamatorio es relevante, tanto en la patogénesis como en las manifestaciones clínicas de la enfermedad ${ }^{3}$.

La EEC no tiene actualmente un tratamiento curativo. No existen estudios controlados, sólo unos pocos ensayos observacionales prospecti$\operatorname{vos}^{1,21}$. En algunas series de casos comunicados en el siglo XX se emplearon corticoides, alcaloides de la vinka, antraciclinas, ciclofosfamida, metotrexato, cladribina, bifosfonatos y trasplante autólogo de células troncales, sin obtener curación ${ }^{3,8}$.

Posteriormente, la utilización de IFN- $\alpha$ se impuso como terapia de primera línea ante la evidencia que producía estabilización de la enfermedad o remisiones parciales y que prolongaba significativamente la sobrevida ${ }^{3,8,9}$. Las dosis habitualmente utilizadas son 3 millones de unidades $(\mathrm{mIU}) / 3$ veces por semana o interferón pegilado
(PEG-IFN) $135 \mathrm{ug} / 1 \mathrm{vez}$ por semana. En los pacientes con compromiso de SNC o cardiovascular estas dosis con frecuencia han sido inefectivas incrementándose hasta $27 \mathrm{mIU} /$ semanales o $>180$ ug/semanales, respectivamente, con respuestas positivas en algunos de ellos ${ }^{3,9}$. Si bien el PEG-IFN es mejor tolerado que el IFN- $\alpha$, frecuentemente se asocian a efectos secundarios como síntomas constitucionales, neurosiquiátricos, digestivos, prurito, alopecía y mielosupresión, que obligan a reducir las dosis o a suspenderlos ${ }^{3}$. Se postula que el IFN- $\alpha$ induce la diferenciación terminal de los histiocitos inmaduros y su muerte ${ }^{21}$. Cuando el IFN- $\alpha$ no ha sido efectivo o tolerado se han empleado otros medicamentos, con respuestas variables.

Anakinra, un anticuerpo recombinante contra el receptor de interleukina-1 se ha utilizado en algunos pacientes, observándose reducción en la masa de la enfermedad y en la concentración de citokinas proinflamatorias. En un caso con compromiso cardiaco el uso de esta droga fue exitoso. Se la ha considerado una terapia razonable en pacientes sin compromiso del SNC y con síntomas constitucionales y óseos. La dosis empleada es $1-2 \mathrm{mg} / \mathrm{kg} /$ día por vía subcutánea. Los efectos secundarios más frecuentes son cefalea, artralgias, nasofaringitis, prurito y eritema cutáneo ${ }^{3,15,16}$.

Infliximab, un anticuerpo anti-TNF- $\alpha$, ha sido utilizado en algunos pacientes con compromiso cardiaco que no respondieron a IFN- $\alpha$. En ellos se observó mejoría clínica y en el compromiso cardiaco con reducción de las citokinas y quemokinas circulantes ${ }^{3,10,13,17}$. La dosis usada fue $5 \mathrm{mg} / \mathrm{kg}$ ev cada 6 semanas. Esta droga produce neutralización del TNF- $\alpha$ soluble y del unido a membranas, disrupción de la cascada de citokinas reguladas por TNF- $\alpha$ y posiblemente lisis de los histiocitos que expresan esta molécula ${ }^{17}$.

Imatinib es un inhibidor competitivo de la tirosin-kinasa, que ha sido utilizado en algunos pacientes con EEC, dada la frecuente expresión en los histiocitos del receptor beta del factor de crecimiento derivado de plaquetas, el cual es inhibido por esta droga. En la mayoría de los pacientes con compromiso cardiovascular se observó estabilización de la enfermedad ${ }^{13,14}$. La dosis promedio empleada fue $350 \mathrm{mg} /$ día (hasta $800 \mathrm{mg} /$ día).

Vemurafenib es un inhibidor del BRAF mutante que ha demostrado eficacia en melanomas y leucemia de células velludas. Recientemente se comunicó su eficacia en 8 casos de EEC refractaria 
a IFN- $\alpha$, en que hubo estabilización o mejoría clínica con reducción significativa en el compromiso periaórtico y en la infiltración cardiaca. La dosis empleada fue $480-960 \mathrm{mg}$ cada $12 \mathrm{~h}$ por vía oral $^{1,3,12,21}$. Los efectos secundarios más frecuentes fueron rash cutáneo, xerosis y queratosis pilaris.

Recientemente fue publicada una experiencia prospectiva con sirolimus en 10 pacientes con un seguimiento promedio de 29 meses. Se obtuvo estabilización de la enfermedad en 8 y progresión en 2. La tolerancia fue buena y la mortalidad fue $20 \%$, inferior a $60 \%$ comunicada en otras series con otras drogas ${ }^{21}$. Los autores concluyen que esta droga pudiera ser utilizada en los pacientes que no tienen la mutación BRAF ${ }^{\mathrm{V} 600 \mathrm{E}}$.

Actualmente hay varios estudios prospectivos en marcha con inhibidores de BRAF (vemurafenib, dabranenib) y con un inhibidor de interleukina 6 (tocilizumab) ${ }^{25-27}$.

Creemos que en este paciente, el uso de sirolimus, produjo una estabilización de su enfermedad cardiaca, permitiéndole sobrevivir 4,5 años adicionales después de un taponamiento cardiaco, en que se comprobó que su corazón estaba difusamente infiltrado por histiocitos. Si bien esta enfermedad es rara, cada vez se describen más casos, probablemente porque ha aumentado su reconocimiento, por lo que parece recomendable conocer que existen tratamientos que permiten prolongar la vida de estos pacientes.

\section{Referencias}

1. Haroche J, Cohen-Aubart F, Emile JF, Maksud P, Drier A, Tolédano D, et al. Reproducible and sustained efficacy of targeted therapy with vemurafenib in patients with BRAF ${ }^{\mathrm{V} 600 \mathrm{E}}$-mutated Erdheim-Chester disease. J Clin Oncol 2015; 33: 411-8.

2. Veyssier-Belot C, Cacoub P, Caparros-Lefrebvre D, Wechsler J, Brun B, Remy M, et al. Erdheim-Chester disease: clinical and radiologic characteristics of 59 cases. Medicine (Baltimore) 1996; 75: 157-69.

3. Diamond EL, Dagna L, Hyman DM, Cavalli G, Janku F, Estrada-Veras J, et al. Consensus guidelines for the diagnosis and clinical management of Erdheim-Chester disease. Blood 2014; 124: 483-92.

4. Brun AL, Touitou-Gottenberg D, Haroche J, Toledano D, Cluzel P, Beigelman-Aubry C, et al. Erdheim-Chester disease: CT findings of thoracic involvement. Eur Radiol 2010; 20: 2579-87.
5. Haroche J, Amoura Z, Dion E, Wechsler B, Costedoat-Chalumeau N, Cacoub P, et al. Cardiovascular involvement, and overlooked feature of Erdheim-Chester disease report of 6 new cases and a literature review. Medicine (Baltimore) 2004; 83: 371-92.

6. Haroche J, Cluzel P, Toledano D, Montalescot G, Touitou $\mathrm{D}$, et al. Images in cardiovascular medicine. Cardiac involvement in Erdheim-Chester disease. Magnetic resonance and computed tomographic scan imaging in a monocentric series of 37 patients. Circulation 2009; 119: e597-e598.

7. Mazor RD, Manevich-Mazor M, Shoenfeld Y. Erdheim-Chester disease: a comprehensive review of the literature. Orphanet J Rare Dis 2013; 8: 137.

8. Arnaud L, Hervier B, Néel A, Hamidou MA, Kahn JE, Wechsler B, et al. CNS involvement and treatment with interferon- $\alpha$ are independent prognostic factors in Erdheim-Chester disease: a multicenter survival analysis of 53 patients. Blood 2011; 117: 2778-82.

9. Hervier B, Arnaud L, Charlotte F, Wechsler B, Piette JC, Amoura Z, et al. Treatment of Erdheim-Chester disease with long-term high-dose interferon- $\alpha$. Semin Arthritis Rheum 2012; 41: 907-13.

10. Ferrero E, Belloni D, Corti A, Doglioni C, Dagna L, Ferrarini M. TNF- $\alpha$ in Erdheim-Chester disease pericardial effusion promotes endotelial leakage in vitro and is neutralized by infliximab. Rheumatology (Oxford) 2014; 53: 198-200.

11. Allen CE, Ladisch S, McClain KL. How I treat Langerhans cell histiocytosis. Blood 2015; 126: 26-35.

12. Haroche J, Cohen-Aubart F, Emile JF, Arnaud L, Maksud P, Charlotte F, et al. Dramatic efficacy of vemurafenib in both multisystemic and refractory Erdheim-Chester disease and Langerhans cell histiocytosis harboring the BRAF ${ }^{\mathrm{6} 60 \mathrm{E}}$ mutation. Blood 2013; 121: 1495-500.

13. Haroche J, Amoura Z, Charlotte F, Salvatierra J, Wechsler B, Graux C, et al. Imatinib mesylate for platelet-derived growth factor receptor-beta-positive Erdheim-Chester histiocytosis. Blood 2008; 111: 5413-5.

14. Janku F, Amin HM, Yang D, Garrido-Laguna I, Trent JC, Kurzrock R. Response of histiocytoses to imatinib mesylate: Fire to ashes. J Clin Oncol 2010; 28: e633-e636.

15. Killu AM, Liang JJ, Jaffe AS. Erdheim-Chester disease with cardiac involvement successfully treated with anakinra. Int J Cardiol 2013; 167: e115-e117.

16. Aouba A, Georgin-Lavialle S, Pagnoux C, Silva NM, Renand A, Galateau-Salle F, et al. Rationale and efficacy of interleukin-1 targeting in Erdheim-Chester disease. Blood 2010; 116: 4070-6.

17. Dagna L, Corti A, Langheim S, Guglielmi B, De Cobelli 
F, Doglioni C, et al. Tumor necrosis factor as a master regulator of inflammation in Erdheim-Chester disease: rationale for the treatment od patients with infliximab. J Clin Oncol 2012; 30: e286-e290.

18. Haroche J, Charlotte F, Arnaud L, von Deimling A, Hélias-Rodzewicz Z, Hervier B, et al. High prevalence of BRAF V600E mutations in Erdheim-Chester disease but not in other non-Langerhans cell histiocytoses. Blood 2012; 120: 2700-3.

19. Graziani G, Podestá MA, Cucchiari D, Reggiani F, Ponticelli C. Erdheim-Chester disease: from palliative care to targeted treatment. Clin Kidney J 2014; 7: 339-43.

20. Myra C, Sloper L, Tighe P, McIntosh RS, Stevens SE, Gregson RH, et al. Treatment of Erdheim-Chester disease with cladribine: a rational approach. Br J Ophthalmol 2004; 88: 844-7.

21. Gianfreda D, Nicastro M, Galetti M, Alberici F, Corradi D, Becchi G, et al. Sirolimus plus prednisone for Erdheim-Chester disease: an open-label trial. Blood 2015; 126: 1163-71.

22. Vega J, Cisternas M, Bergoeing M, Espinosa R, Zapico A, Chadid P, et al. Enfermedad de Erdheim-Chester. Una causa rara de derrame pericárdico. Caso clínico. Rev Med Chile 2011; 139: 1054-9.
23. Vaglio A, Casazza I, Alberici F, Palmisano A, Foti R, Moroni $G$, et al. Sirolimus plus prednisone for the treatment of Erdheim-Chester disease. A pilot study. Presentado en World Congress of Nephrology, Milan, mayo 22-26, 2009.

24. Arnaud L, Gorochov G, Charlotte F, Lvovschi V, Parizot C, Larsen M, et al. Systemic perturbation of cytokine and chemokine networks in Erdheim-Chester disease: a single-center series of 37 patients. Blood 2011; 117: 2783-90.

25. Long-term Outcome After Vemurafenib/BRAF Inhibitors Interruption in Erdheim-chester Disease (LOVE). Accedido el 29 de septiembre de 2015 en: https://www. clinicaltrials.gov/ct2/show/NCT02089724?term=Erdheim-Chester\&rank=2.

26. Dabrafenib and Trametinib in People With BRAF V600E Mutation Positive Lesions in Erdheim Chester Disease. Accedido el 29 de septiembre de 2015 en: https://www.clinicaltrials.gov/ct2/show/NCT02281760?term $=$ Erdheim-Chester\&rank $=4$.

27. Pilot Study of Tocilizumab in Patients With Erdheim-Chester Disease. Accedido el 29 de septiembre de 2015 en: https://www.clinicaltrials.gov/ct2/show/ NCT01727206?term=Erdheim-Chester\&rank=1. 